\title{
GOAL-ORIENTED ERROR ESTIMATION AND MESH AdAPTATION FOR SHALLOW WATER MODELLING
}

A NON-PEER REVIEWED PREPRINT SUBMITTED TO EARTHARXIV

\author{
Joseph G. Wallwork \\ Department of Earth Science and Engineering \\ Imperial College London, UK \\ j.wallwork16@imperial.ac.uk \\ Stephan C. Kramer \\ Department of Earth Science and Engineering \\ Imperial College London, UK
}

\author{
Nicolas Barral \\ University of Bordeaux, CNRS, Bordeaux INP \\ IMB, UMR 5251, 33400, Talence, France \\ INRIA, IMB, UMR 5251, 33400, Talence, France
}

\author{
David A. Ham \\ Department of Mathematics \\ Imperial College London, UK
}

\author{
Matthew D. Piggott \\ Department of Earth Science and Engineering \\ Imperial College London, UK
}

July 28,2020

\begin{abstract}
Numerical modelling frequently involves a diagnostic quantity of interest (QoI) - often of greater importance than the PDE solution - which we seek to accurately approximate. In the case of coastal ocean modelling the power output of a tidal turbine farm is one such example. Goal-oriented error estimation and mesh adaptation can be used to provide meshes which are well-suited to achieving this goal, using fewer computational resources than required by other methods, such as uniform refinement.

A mixed discontinuous/continuous Galerkin approach is applied to solve the nonlinear shallow water equations within the Thetis coastal ocean finite element model [1]. An implementation of goal-oriented mesh adaptation is outlined, including an error estimate which takes account of the discontinuities in the discrete solution and a method for approximating the adjoint error. Results are presented for simulations of two model tidal farm configurations. Convergence analysis indicates that the anisotropic goal-oriented adaptation strategy yields meshes which permit accurate QoI estimation using fewer computational resources than uniform refinement.
\end{abstract}

Keywords Adjoint methods · Mesh adaptation · Discontinuous Galerkin · Tidal Turbine Modelling · Firedrake

\section{Introduction}

The depth-averaged shallow water equations are often used in coastal ocean models, providing approximations to the fluid velocity and elevation of the ocean surface. This work utilises the Thetis coastal ocean modelling framework [1], which is based upon the finite element library Firedrake [2]. As well as a 2D shallow water model [3], Thetis offers 3D Navier-Stokes solvers with Boussinesq and hydrostatic [1] and non-hydrostatic [4] assumptions and a tracer transport model.

Coastal modelling problems are typically multi-scale, often with a strongly direction-dependent flow. As such, anisotropic mesh adaptation is an attractive prospect for providing both accuracy and reduced computational cost; by adapting the mesh such that its anisotropy is aligned with the flow, the number of degrees of freedom (DOFs) required to 
yield an accurate solution is reduced. The metric based approach to anisotropic mesh adaptation was first introduced in [5] and uses Riemannian metric fields to control not only the size of mesh elements, but also their shape and orientation. This approach was shown to be particularly suited to multi-scale ocean modelling in [6].

Further, coastal modelling problems often involve a diagnostic quantity of interest (QoI) of greater importance than the solution itself. Goal-oriented error estimation represents the error accrued in computing the QoI in terms of PDE residuals and solutions of associated adjoint equations. Used within a mesh adaptation algorithm, such estimators can yield meshes permitting accurate QoI approximation. The majority of goal-oriented error estimators are based upon the pioneering work of [7, 8]. More recently, researchers have developed goal-oriented estimators which take account of discontinuous Galerkin discretisations (see [9, 10]). Integration into the metric based mesh adaptation framework has also been developed (see [11, 12, 13]).

This work builds upon the research referenced above, focusing on the shallow water equations. To the best of the authors' knowledge, it presents the first formulation of a goal-oriented error estimate for these equations discretised in a mixed discontinuous/continuous space.

Goal-oriented error estimation is introduced in Section 2, along with two approaches to goal-oriented mesh adaptation. Application of the error estimation and adaptation techniques to shallow water problems is considered in Section 3 Section 4 contains numerical experiments validating the adaptation strategy for a simple tidal turbine model. Conclusions are drawn in Section 5

\section{Goal-Oriented Mesh Adaptation}

\subsection{Metric-Based Mesh Adaptation}

In this paper, Riemannian metric fields are used to drive the mesh adaptation process. These tensor fields, often referred to simply as metrics, are symmetric positive-definite (SPD) linear forms defined pointwise, which give rise to all of the geometrical quantities necessary to perform mesh adaptation. In this work, metrics are defined using the error estimates described in Subsection 2.2 For details on the metric-based approach, see [5, 14, 15, 16].

The spatial domain, denoted by $\Omega \subset \mathbb{R}^{n}$, is assumed to have piecewise smooth boundary $\Gamma:=\partial \Omega$. For a mesh $\mathcal{H}$ of the domain, we denote mesh elements by $K \in \mathcal{H}$ and the edge set of element $K$ by $\partial K$. We denote the set of all edges which are not on the domain boundary (internal edges) by $\Gamma_{\text {int }}$.

\subsection{Error Estimation}

The shallow water equations may be written in the form

$$
\Psi(\mathbf{q})=\mathbf{0}
$$

with solution q living in a space of functions denoted $V$. Throughout this paper, we shall refer to (1) as the forward equation and to $\mathbf{q}$ as the forward solution. The diagnostic QoI, $J$, is a functional which maps members of $V$ onto the real number line. Associated with (1) and the QoI is an adjoint equation,

$$
\Psi^{*}\left(\mathbf{q}^{*}\right)=\frac{\partial J}{\partial \mathbf{q}} .
$$

The adjoint solution $\mathbf{q}^{*}$ also lives in $V$ and conveys the sensitivities of the QoI to perturbations in the forward solution. For a finite dimensional subspace $V_{h} \subset V$, we have Galerkin approximations to (1) and (2) given by

$$
\begin{aligned}
\rho\left(\mathbf{q}_{h}, \boldsymbol{\xi}\right)=-\left\langle\boldsymbol{\Psi}\left(\mathbf{q}_{h}\right), \boldsymbol{\xi}\right\rangle=0, & \forall \boldsymbol{\xi} \in V_{h}, \\
\rho^{*}\left(\mathbf{q}_{h}^{*}, \boldsymbol{\xi}\right)=\left\langle\frac{\partial J}{\partial \mathbf{q}}-\boldsymbol{\Psi}^{*}\left(\mathbf{q}_{h}^{*}\right), \boldsymbol{\xi}\right\rangle=0, & \forall \boldsymbol{\xi} \in V_{h},
\end{aligned}
$$

where $\langle\cdot, \cdot\rangle$ is the $\mathcal{L}_{2}$ inner product. Typically, integration by parts is applied in constructing the weak residuals $\rho\left(\mathbf{q}_{h}, \cdot\right)$ and $\rho^{*}\left(\mathbf{q}_{h}^{*}, \cdot\right)$ from the inner products.

We consider the classical a posteriori goal-oriented error estimate known as the dual weighted residual, due to [7, 8]. This error estimate is given by

$$
J(\mathbf{q})-J\left(\mathbf{q}_{h}\right)=\rho\left(\mathbf{q}_{h}, \mathbf{q}^{*}-\mathbf{q}_{h}^{*}\right)+R^{(2)}
$$

where the remainder term $R^{(2)}$ is quadratic in the forward and adjoint errors $\mathbf{q}-\mathbf{q}_{h}$ and $\mathbf{q}^{*}-\mathbf{q}_{h}^{*}$. Element-wise error indicators and a global error estimator may be derived as

$$
\mathcal{E}_{K}=\left|\rho\left(\mathbf{q}_{h}, \mathbf{q}^{*}-\mathbf{q}_{h}^{*}\right)\right|_{K} \mid, \quad \mathcal{E}=\sum_{K \in \mathcal{H}} \mathcal{E}_{K} .
$$




\subsection{Goal-Oriented Metrics}

There are many potential ways to construct metric tensor fields using the scalar error indicator (6), one of which is to appropriately scale an identity matrix; this is referred to as the isotropic case, since resulting meshes are relatively isotropic. For problems with strong directional dependence, anisotropic metrics can be beneficial, allowing control of the shape and orientation of mesh elements, as well as size.

In [17], an isotropic metric was compared with two approaches to constructing anisotropic metrics from goal-oriented error estimates (based on the work of [11] and [12]) for advection-diffusion problems discretised using continuous finite elements. In the remainder of this subsection, we utilise a different approach, developed in [13], which straightforwardly permits discontinuous discretisations.

\subsubsection{Isotropic Metric}

First, consider the isotropic case. Let $|\widehat{K}|$ denote the reference element volume and $|K|$ denote the volume of an arbitrary element $K \in \mathcal{H}$. Constructing an element-based isotropic metric in $n$-dimensions amounts to the scaling [13]

$$
\overline{\mathcal{M}}_{K}=\frac{|\widehat{K}|}{|\widetilde{K}|} \mathcal{I}_{n}, \quad|\widetilde{K}|=|K|\left(\frac{\sum_{K \in \mathcal{H}} \mathcal{E}_{K}^{\frac{1}{\alpha+1}}}{N}\right) \mathcal{E}_{K}^{-\frac{1}{\alpha+1}}
$$

where $\mathcal{I}_{n}$ is the element-wise identity metric. The desired element volume $|\widetilde{K}|$ is chosen to minimise interpolation error, subject to a desired metric complexity $N>0$ [13]. Metric complexity can be viewed as the continuous analogue of the element count of a mesh [15]. The parameter $\alpha \geq 1$ which arises in the solution of this optimisation problem is not known a priori. However, it is stated in [13] that its influence on (7) is negligible, provided that we are sufficiently close to the optimal element size. We have found $\alpha=1$ to be an effective choice in practice.

\subsubsection{Anisotropic Metric}

As with the approaches considered in [17], the anisotropic metric construction of [13] uses a recovered Hessian of the prognostic variables. In this instance, we compute the element-averaged Hessian $\overline{H_{K}}$ on an element $K$ by solving an auxiliary finite element problem (for details, see [14]).

As a symmetric matrix, the Hessian has an orthogonal eigen-decomposition $\overline{H_{K}}=V_{K} \Lambda_{K} V_{K}^{T}$, with eigenvalue matrix $\Lambda_{K}=\operatorname{diag}\left(\lambda_{K, 1}, \ldots, \lambda_{K, n}\right)$ ordered such that $\left|\lambda_{K, 1}\right| \leq \cdots \leq\left|\lambda_{K, n}\right|$. The stretching factor associated with the element-averaged Hessian is defined by

$$
s_{K}:=\sqrt{\frac{\max _{i=1}^{n}\left|\lambda_{i, K}\right|}{\min _{i=1}^{n}\left|\lambda_{i, K}\right|}} .
$$

We construct an element-wise metric by modifying the eigenvalues appropriately. In the two dimensional case, [13]

$$
\overline{\mathcal{M}_{K}}=V_{K} \widetilde{\Lambda}_{K}^{-2} V_{K}^{T}, \quad \widetilde{\Lambda}_{K}=\operatorname{diag}\left(\sqrt{\frac{|\widetilde{K}|}{|\widehat{K}|} s_{K}}, \sqrt{\frac{|\widetilde{K}|}{|\widehat{K}|} \frac{1}{s_{K}}}\right)
$$

A vertex-based metric is obtained by projection, using $\Pi_{\mathbb{P} 1}: \mathbb{P} 0 \rightarrow \mathbb{P} 1$.

\section{Application to the Shallow Water Equations}

\subsection{Shallow Water Equations}

In this work we consider the steady-state form of the nonlinear shallow water equations,

$$
\mathbf{u} \cdot \nabla \mathbf{u}+g \nabla \eta+\frac{C_{d}\|\mathbf{u}\| \mathbf{u}}{H}=\nabla \cdot(D \nabla \mathbf{u}), \quad \nabla \cdot(H \mathbf{u})=0,
$$

for velocity $\mathbf{u}$ and surface elevation $\eta$, with boundary conditions as appropriate. The fluid is modelled as having viscosity tensor $D$, quadratic drag $C_{d}$ and bathymetry $b$, giving rise to a total depth $H=b+\eta$. We assume $g=9.81 \mathrm{~m} \mathrm{~s}^{-2}$.

Suppose the exact solution $\mathbf{q}=(\mathbf{u}, \eta)$ lives in a function space $V$ with finite dimensional subspace $V_{h}$. For all test functions $\boldsymbol{\xi}=(\boldsymbol{\psi}, \phi) \in V_{h}$, we have a Galerkin formulation of 10 given by

$$
\rho_{\mathrm{adv}}\left(\mathbf{q}_{h}, \boldsymbol{\xi}\right)+\rho_{\mathrm{gra}}\left(\mathbf{q}_{h}, \boldsymbol{\xi}\right)+\rho_{\mathrm{vis}}\left(\mathbf{q}_{h}, \boldsymbol{\xi}\right)+\rho_{\mathrm{drg}}\left(\mathbf{q}_{h}, \boldsymbol{\xi}\right)+\rho_{\mathrm{cty}}\left(\mathbf{q}_{h}, \boldsymbol{\xi}\right)=0,
$$


where $\mathbf{q}_{h} \in V_{h}$ is a finite element approximation to $\mathbf{q}$. The weak form is decomposed into advection, gravity, viscosity, drag and continuity terms. Taking all test functions, $\sqrt{11}$ ) forms a nonlinear system of equations which is solved using a Newton iteration. A direct solver is used in this work to solve the linear system of equations that arises in the update step of Newton's method based on the Jacobian of (11).

For $V_{h}$, we select the mixed finite element space $\mathbb{P} 1_{D G}-\mathbb{P} 2$. That is, velocity is piecewise linear and discontinuous across elemental boundaries, whilst elevation is piecewise quadratic and continuous. This element pair was shown to be suitable for shallow water modelling in [18].

Clearly, functions $v$ from the velocity space are discontinuous across internal edges. Thus, internal edge integrals whose integrand involves $v$ are not well defined. This necessitates the introduction of the following restriction operators, which assign a single value on all edges. For an edge $\gamma \in \Gamma_{\text {int }}$, arbitrarily label each side with + and - , giving rise to normal vectors $\widehat{\mathbf{n}}^{ \pm}$. Define the restriction operators given by the average $\left\{\left.\{v\}\right|_{\gamma}:=\frac{1}{2}\left(v^{+}+v^{-}\right)\right.$and jump $\left.\llbracket v \cdot \widehat{\mathbf{n}} \rrbracket\right|_{\gamma}:=v^{+} \cdot \widehat{\mathbf{n}}^{+}+v^{-} \cdot \widehat{\mathbf{n}}^{-}$. For edges on the domain boundary, set both the average and jump to the function value on the boundary. Denote the outer product $\mathbf{v}_{1} \mathbf{v}_{2}^{T}$ of two vector functions by $\mathbf{v}_{1} \otimes \mathbf{v}_{2}$.

Ignoring boundary condition implementation (for brevity), Thetis uses the discretisation,

$$
\begin{aligned}
\rho_{\mathrm{adv}}\left(\mathbf{q}_{h}, \boldsymbol{\xi}\right) & =-\int_{\Omega}\left(\nabla \cdot\left(\mathbf{u}_{h} \otimes \boldsymbol{\psi}\right)\right) \cdot \mathbf{u}_{h} \mathrm{~d} x+\int_{\Gamma_{\mathrm{int}}} \llbracket \boldsymbol{\psi}\left(\mathbf{u}_{h} \cdot \widehat{\mathbf{n}}\right) \rrbracket \cdot\left\{\left\{\mathbf{u}_{h}\right\} \mathrm{d} S+\int_{\Gamma_{\mathrm{int}}} \tau \llbracket \boldsymbol{\psi} \rrbracket \cdot \llbracket \mathbf{u}_{h} \rrbracket \mathrm{d} S,\right. \\
\rho_{\mathrm{gra}}\left(\mathbf{q}_{h}, \boldsymbol{\xi}\right) & =\int_{\Omega} g \boldsymbol{\psi} \cdot \nabla \eta_{h} \mathrm{~d} x, \\
\rho_{\mathrm{vis}}\left(\mathbf{q}_{h}, \boldsymbol{\xi}\right) & =\int_{\Omega} \nabla \boldsymbol{\psi}: D \nabla \mathbf{u}_{h} \mathrm{~d} x+\int_{\Gamma_{\mathrm{int}}} \sigma \llbracket \boldsymbol{\psi} \otimes \widehat{\mathbf{n}} \rrbracket:\left\{\{D\} \llbracket \mathbf{u}_{h} \otimes \widehat{\mathbf{n}} \rrbracket \mathrm{d} S\right. \\
- & \int_{\Gamma_{\mathrm{int}}} \llbracket \boldsymbol{\psi} \otimes \widehat{\mathbf{n}} \rrbracket:\left\{\left\{D \nabla \mathbf{u}_{h}\right\} \mathrm{d} S-\int_{\Gamma_{\mathrm{int}}}\left\{\{\nabla \boldsymbol{\psi}\}:\left\{\{D\} \llbracket \mathbf{u}_{h} \otimes \widehat{\mathbf{n}} \rrbracket \mathrm{d} S,\right.\right.\right. \\
\rho_{\mathrm{drg}}\left(\mathbf{q}_{h}, \boldsymbol{\xi}\right) & =\int_{\Omega} \boldsymbol{\psi} \cdot \frac{C_{d}\left\|\mathbf{u}_{h}\right\| \mathbf{u}_{h}}{H_{h} \mathrm{~d} x,} \\
\rho_{\mathrm{cty}}\left(\mathbf{q}_{h}, \boldsymbol{\xi}\right) & =-\int_{\Omega} \nabla \phi \cdot\left(H_{h} \mathbf{u}_{h}\right) \mathrm{d} x,
\end{aligned}
$$

where $H_{h}:=b+\eta_{h}$. If the elevation was chosen from a discontinuous space (which is also a valid choice in Thetis) then the gravity term would be integrated by parts and we would need Riemann solutions for $\mathbf{u}$ and $\eta$.

Lax-Friedrichs stabilisation [19] is applied in the advection term with parameter $\tau=\frac{1}{2}\left|\{\{\mathbf{u}\}\} \cdot \widehat{\mathbf{n}}^{+}\right|$. The interior penalty parameter $\sigma$ used in the viscous term is chosen in line with [20], depending upon the polynomial degree, variation of $D$ and the minimal angle in each mesh element. For simplicity, we set $\sigma$ as the largest element-wise value. It is important to update the penalty parameter whenever the mesh is adapted. Boundary conditions are imposed weakly, therefore contributing additional terms to $[12)$.

\subsection{Goal-Oriented Error Estimate}

As discussed in Subsection 2.2, the construction of goal-oriented error estimators from (12) amounts to integrating by parts on each element and substituting test functions for the adjoint error $\mathbf{e}^{*}=\mathbf{q}^{*}-\mathbf{q}_{h}^{*}$, which appears in (5)-(6). Here $\mathbf{q}^{*}=\left(\mathbf{u}^{*}, \eta^{*}\right)$ is the exact adjoint solution, $\mathbf{q}_{h}^{*}=\left(\mathbf{u}_{h}^{*}, \eta_{h}^{*}\right)$ is a finite element approximation thereof and $\mathbf{e}^{*}=\left(\mathbf{e}_{\mathbf{u}}^{*}, e_{\eta}^{*}\right)$ is the corresponding error. No integration by parts is required for the gravity or drag terms; the test function is simply substituted for the adjoint error. We seek element-based error indicators, so there is no need to restrict quantities which arise. Following the approach of [9], we write adjoint variables as represented on each element. 
The following terms of the goal-oriented error indicator arise, following integration by parts:

$$
\begin{gathered}
\left.\rho_{\text {adv }}\left(\mathbf{q}_{h}, \mathbf{e}^{*}\right)\right|_{K}=\quad \int_{K} \mathbf{e}_{\mathbf{u}}^{*} \cdot\left(\mathbf{u}_{h} \cdot \nabla \mathbf{u}_{h}\right) \mathrm{d} x-\int_{\partial K}\left(\left(\mathbf{u}_{h} \otimes \mathbf{e}_{\mathbf{u}}^{*}\right) \cdot \mathbf{u}_{h}\right) \cdot \widehat{\mathbf{n}} \mathrm{d} S, \\
+\int_{\partial K \backslash \Gamma} \llbracket \mathbf{u}_{h} \cdot \widehat{\mathbf{n}} \rrbracket \mathbf{e}_{\mathbf{u}}^{*} \cdot\left\{\left\{\mathbf{u}_{h}\right\}\right\} \mathrm{d} S+\int_{\partial K \backslash \Gamma} \tau \llbracket \mathbf{e}_{\mathbf{u}}^{*} \rrbracket \cdot \llbracket \mathbf{u} \rrbracket \mathrm{d} S, \\
\left.\rho_{\text {vis }}\left(\mathbf{q}_{h}, \mathbf{e}^{*}\right)\right|_{K}=\int_{K} \mathbf{e}_{\mathbf{u}}^{*} \cdot\left(\nabla \cdot\left(D \nabla \mathbf{u}_{h}\right)\right) \mathrm{d} x-\int_{\partial K} \mathbf{e}_{\mathbf{u}}^{*} \otimes \widehat{\mathbf{n}}: D \nabla \mathbf{u}_{h} \mathrm{~d} S \\
-\int_{\partial K \backslash \Gamma} \sigma \mathbf{e}_{\mathbf{u}}^{*} \otimes \widehat{\mathbf{n}}:\left\{\{D\} \llbracket \llbracket \mathbf{u}_{h} \otimes \widehat{\mathbf{n}} \rrbracket \mathrm{d} S\right. \\
+\int_{\partial K \backslash \Gamma} \mathbf{e}_{\mathbf{u}}^{*} \otimes \widehat{\mathbf{n}}:\left\{\left\{D \nabla \mathbf{u}_{h}\right\}\right\} \mathrm{d} S+\frac{1}{2} \int_{\partial K \backslash \Gamma} \nabla \mathbf{e}_{\mathbf{u}}^{*}:\left\{\{D\} \llbracket \llbracket \mathbf{u}_{h} \otimes \widehat{\mathbf{n}} \rrbracket \mathrm{d} S\right. \\
\left.\rho_{\text {cty }}\left(\mathbf{q}_{h}, \mathbf{e}^{*}\right)\right|_{K}=\int_{K} e_{\eta}^{*} \nabla \cdot\left(H_{h} \mathbf{u}_{h}\right) \mathrm{d} x-\int_{\partial K} e_{\eta}^{*} H_{h} \mathbf{u}_{h} \cdot \widehat{\mathbf{n}} \mathrm{d} S .
\end{gathered}
$$

The additional boundary terms not shown here assess the extent to which the boundary conditions have been weakly enforced.

The element-wise error indicator is established from (11)-(13) using (6). Note that (13) contains the (unknown) exact adjoint solution, $\mathbf{q}^{*}$. In practice, it suffices to approximate it in an enriched space, $V_{h}^{+} \supset V_{h}$. We again choose $V_{h}^{+}$ as $\mathbb{P} 1_{D G}-\mathbb{P} 2$, but defined on a uniformly refined mesh. That is, a single, global iso- $\mathbb{P} 2$ refinement is made, which amounts to inserting vertices wherever a quadrature node would exist in a quadratic element. The adjoint equation is then derived using a linearisation about the projected forward solution, $\Pi_{h}^{+} \mathbf{q}_{h} \in V_{h}^{+}$. Whilst this approach is shown to be effective in Subsection 4.3 , it implies an additional computational cost associated with solving the adjoint equation on a mesh with four times as many elements. In future work, a more efficient approach will be implemented, such as the one described on pp.590-593 of [10], which solves local PDEs to approximate $\mathbf{q}^{*}$.

\subsection{Implementation Details}

The anisotropic metric formulation described in Subsection 2.3 relies on the provision of an approximate Hessian. Hessian recovery techniques typically seek second derivatives for scalar fields. In this case, there are a number of options for fields to recover a Hessian from - the free surface elevation, velocity components and the fluid speed being the most obvious candidates. Individual Hessians may be combined, using a strategy such as metric averaging or metric superposition (see pp.131-138 of [15] for details and an investigation of the differences). In this work, we superpose the free surface elevation and velocity component Hessians, so that the anisotropy of each field is accounted for.

Mesh adaptation is performed using Pragmatic [16, 21], which is a mesh optimisation library primarily performing $h$-like adaptive operations, with some local Laplacian smoothing. The mesh adaptation workflow used is identical to that described in Algorithm 1 of [17]. The adaptation loop is terminated if the QoI value, number of mesh elements or error estimator 13 change by less than $0.1 \%$ from one iteration to the next.

Previously published studies, such as [22], use the dolfin-adjoint package [23], which automates the solution of the discrete adjoint of a finite element problem expressed in Unified Form Language (UFL) [24]. In this work, we wish to be able to discretise the adjoint problem in a different way than the forward problem. Thus, we exploit the automatic differentiation capabilities of UFL in order to avoid an error-prone manual calculation.

Given a weak form PDE ' $F==0$ ' with finite element solution ' $q$ ' and a QoI ' $J$ ', only a few lines of code are required to compute the adjoint solution, 'q_star':

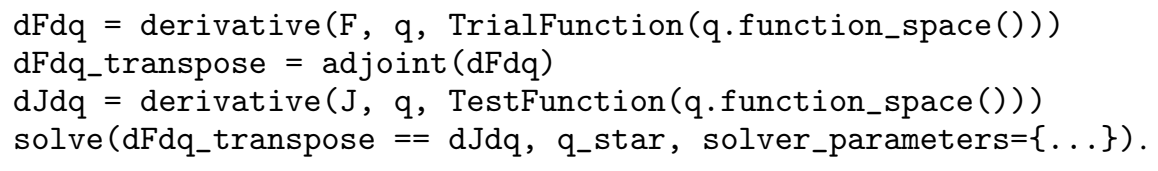

Writing the adjoint equation using Firedrake solve calls enables us to solve the adjoint equation in $V_{h}^{+}$, as opposed to $V_{h}$. To do this, we replace q by $\mathrm{q}_{-} \in V_{h}^{+}$and $\mathrm{F}$ by $\mathrm{F}_{-}$in the above, where $\mathrm{F}_{-}$is defined by prolonging the variables used in $\mathrm{F}$ from $V_{h}$ to $V_{h}^{+}$.

The Firedrake installation used for all simulations documented in Section 4 is archived at [25, 26], with all simulation code archived at [27]. 


\section{Numerical Experiments}

\subsection{Tidal Turbine Modelling}

Marine renewable energy is an active area of research in coastal ocean modelling (for example, see [22, 28, 3, 29, 30]). In particular, tidal power presents an opportunity to generate large amounts of clean electricity in coastal countries, such as the UK. One advantage of tidal power over other renewable energy sources is that tides are highly predictable, meaning that power is generated reliably.

The shallow water depth in which tidal turbines are deployed has a significant impact on wake recovery and hydrodynamic blockage effects, meaning that the positioning of turbines can be very important. By modifying the shallow water equations to account for tidal turbines positioned within the domain, recent research formulated tidal array positioning as a PDE-constrained optimisation problem [22]. Solving this problem gives the configuration with maximum power, with the potential to incorporate penalties in the optimisation functional to account for financial [29] and environmental impact factors [30]. A parametrisation based approach is used, meaning that the turbines are modelled using a density function $d=d(\mathbf{x})$.

Modifying (10) to account for a set of tidal turbines $\mathcal{T}$ amounts to choosing an appropriate drag coefficient $C_{d}$. Suppose turbine $T$ has thrust coefficient $c_{T}$, area $A_{T}$ and footprint indicated by $\mathbb{1}_{T}$. For a background drag $C_{b}=0.0025$,

$$
C_{d}:=C_{b}+C_{t}, \quad C_{t}:=\sum_{T \in \mathcal{T}} \frac{1}{2} d c_{T} A_{T} \mathbb{1}_{T} .
$$

The thrust coefficent used in (14) is based on an upstream velocity, whereas $\mathbf{u}$ is the depth-averaged velocity at turbine $T$. Hence, we correct the thrust coefficient using the rescaling recommended in [31].

We use the following proxy for the power output of the tidal array:

$$
J(\mathbf{u}, \eta):=\int_{\Omega} C_{t}\|\mathbf{u}\|^{3} \mathrm{~d} x .
$$

This provides a QoI for goal-oriented error estimation and has units of Watts.

\subsection{Problem Setup}

Whilst realistic tidal turbine applications are inherently time-dependent, we consider a steady-state test case to highlight the impact of the turbine position on the power output. For a simple tidal farm with two turbines, we consider two configurations: one where $T_{2}$ is directly downstream of $T_{1}$ and one where the turbines are offset so that this is not the case. We apply the goal-oriented mesh adaptation approaches described in Section 2 to provide accurate approximations to the power output of the tidal farm in each case.

For a channel domain $\Omega=\left[0, \ell_{1}\right] \times\left[0, \ell_{2}\right]$ with $\ell_{1}=1.2 \mathrm{~km}$ and $\ell_{2}=500 \mathrm{~m}$, flow is driven by an inflow condition $\left.\mathbf{u}\right|_{x=0}=(5,0) \mathrm{m} \mathrm{s}^{-1}$. Free-slip conditions are imposed on the channel walls, along with a Dirichlet condition $\left.\eta\right|_{x=\ell_{1}}=0 \mathrm{~m}$ on the outflow. Turbines of diameter $18 \mathrm{~m}$ are centred at $\{(456,250),(744,250)\}$ in the aligned case and $\{(356,232),(744,218)\}$ in the offset case. We use $J_{1}$ and $J_{2}$ to denote the total power output 15 for each of the two array configurations considered.

First, we verify that each QoI converges on a sequence of increasingly refined meshes. Coarse initial meshes which take account of the tidal turbines are generated using gmsh [32] and shown in Subfigures $1 \mathrm{a}-1 \mathrm{~b}$. A mesh hierarchy is obtained by iso- $\mathbb{P} 2$ refinement.

\begin{tabular}{|ccc||ccc|}
\hline \multicolumn{3}{c|}{ Aligned } & \multicolumn{3}{c|}{ Offset } \\
Elements & DOFs & $J_{1}$ & Elements & DOFs & $J_{2}$ \\
\hline 3,328 & 26,711 & $20.7460 \mathrm{~kW}$ & 3,170 & 25,447 & $24.1583 \mathrm{~kW}$ \\
13,312 & 106,669 & $19.9454 \mathrm{~kW}$ & 12,680 & 101,613 & $23.4034 \mathrm{~kW}$ \\
53,248 & 426,329 & $19.7451 \mathrm{~kW}$ & 50,720 & 406,105 & $23.2172 \mathrm{~kW}$ \\
212,992 & $1,704,625$ & $19.7201 \mathrm{~kW}$ & 202,880 & $1,623,729$ & $23.1923 \mathrm{~kW}$ \\
851,968 & $6,817,121$ & $19.7174 \mathrm{~kW}$ & 811,520 & $6,493,537$ & $23.1905 \mathrm{~kW}$ \\
\hline
\end{tabular}

Table 1: Convergence of QoIs $J_{1}$ and $J_{2}$ evaluated at finite element solutions on a sequence of meshes generated by uniform refinement of the initial mesh.

Table 1 illustrates the convergence of QoI values under uniform refinement. Final values, $J_{1}=19.7174 \mathrm{~kW}$ and $J_{2}=23.1905 \mathrm{~kW}$, present benchmark values to approximate using adaptive meshes. Note that the converged power 


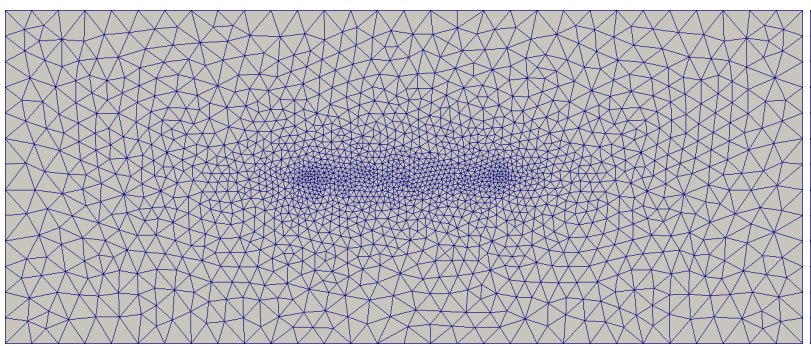

(a) Initial mesh (3,328 elements, max. aspect ratio 1.8)

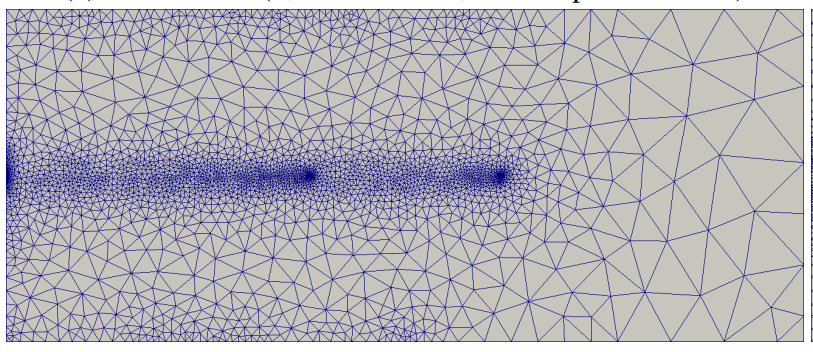

(c) Isotropic adaptation (5,508 elements, max. aspect ratio 1.7 , QoI $20.2358 \mathrm{~kW}$ )

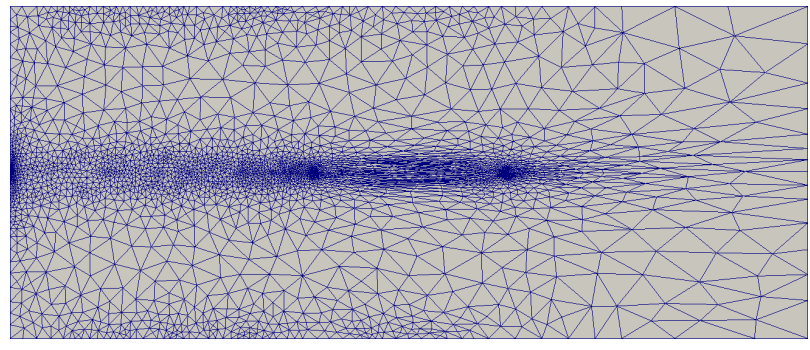

(e) Anisotropic adaptation (6,060 elements, max. aspect ratio 18.3, QoI $19.8468 \mathrm{~kW}$ )

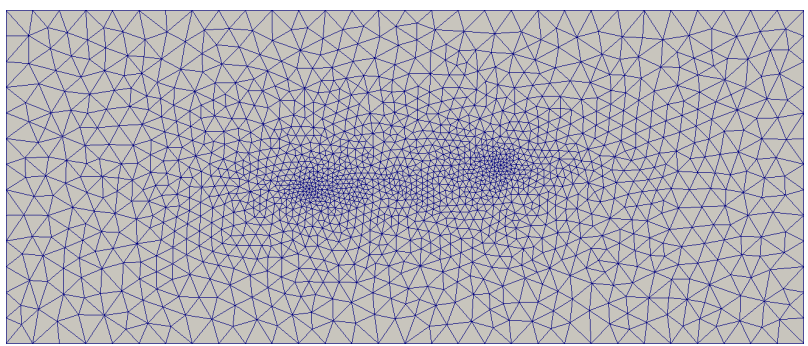

(b) Initial mesh (3,170 elements, max. aspect ratio 1.7)

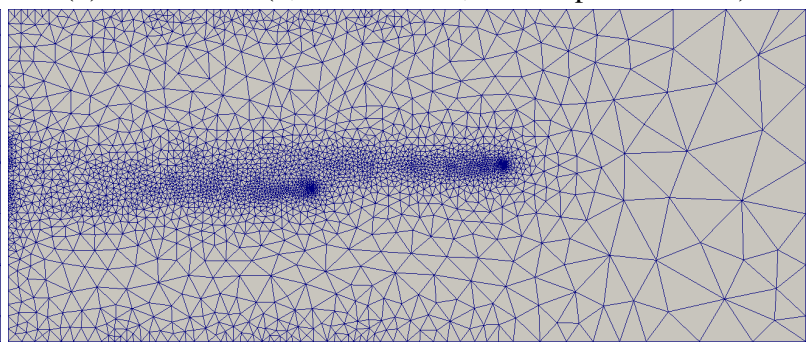

(d) Isotropic adaptation (5,472 elements, max. aspect ratio 2.2, QoI $23.5522 \mathrm{~kW}$ )

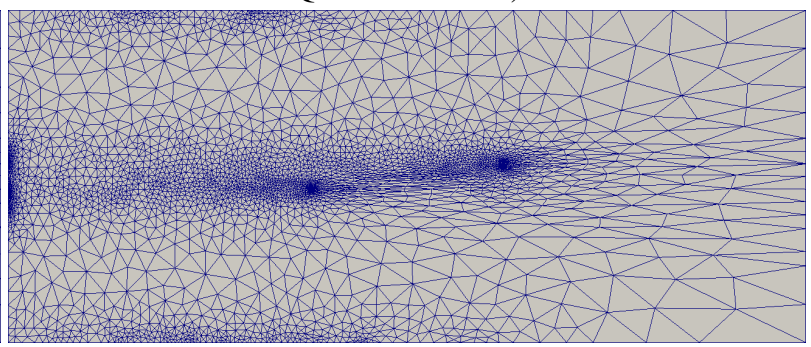

(f) Anisotropic adaptation (6,442 elements, max. aspect ratio 8.7, QoI $23.3632 \mathrm{~kW}$ )

Figure 1: Initial meshes, along with example meshes generated using goal-oriented mesh adaptation. Subfigures $1 \mathrm{a}$ 1c and $1 \mathrm{e}$ correspond to the aligned configuration, whilst Subfigures $1 \mathrm{~b}, 1 \mathrm{~d}$ and $1 \mathrm{f}$ correspond to the offset configuration.

output in the aligned configuration is around $15 \%$ lower than in the offset case. This is due to the downstream turbine being fully in the wake of the upstream turbine in the aligned cased and thus overall experiencing slower flow and consequently generating less power. This illustrates how the positioning of turbines within an array can significantly impact power output.

\subsection{Convergence Analysis under Goal-Oriented Mesh Adaptation}

Having obtained benchmark values for the power output under uniform refinement in each turbine configuration, we apply mesh adaptation in an attempt to achieve convergence to these values using fewer DOFs.

Figure 1 shows meshes generated by each adaptation strategy, each with low resolution downstream of the second turbine. This should be expected since we have an advection-dominated problem and the power output of the array is independent of the downstream dynamics. We observe high mesh resolution surrounding the turbines and in a band between them. The moderate mesh resolution near the domain boundaries in the tidal farm and upstream regions is due to residual contributions from the weakly enforced boundary conditions. As expected, Subfigures $1 \mathrm{e}-1 \mathrm{f}$ contain more anisotropy than Subfigures $1 \mathrm{c}-1 \mathrm{~d}$. For both the aligned and offset configurations, we observe that the anisotropic mesh yields a better approximation of the respective benchmark power output using fewer elements.

For a given number of DOFs, Figure 2 illustrates that both isotropic and anisotropic goal-oriented adaptation yield more accurate power output estimates than uniform refinement of the initial mesh. Further, both adaptation strategies provide sequences of meshes upon which QoI evaluations converge to the benchmark values established in Table 1 . This validates the adaptive solution strategies. 


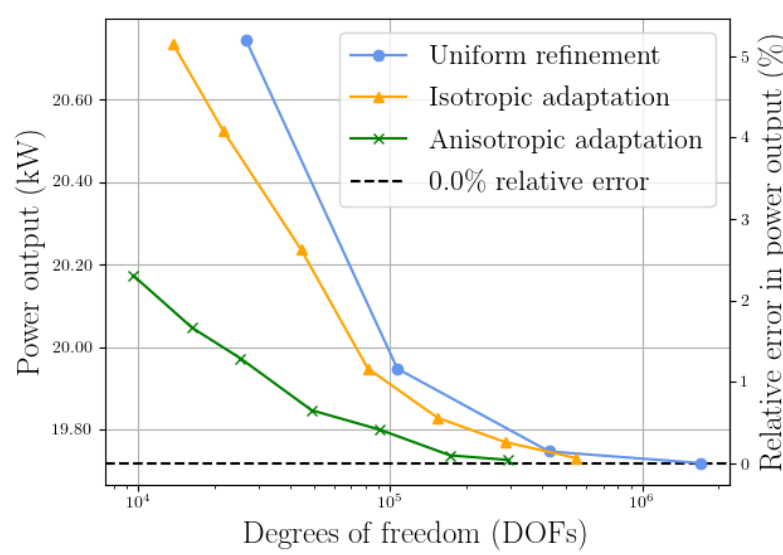

(a) Aligned configuration

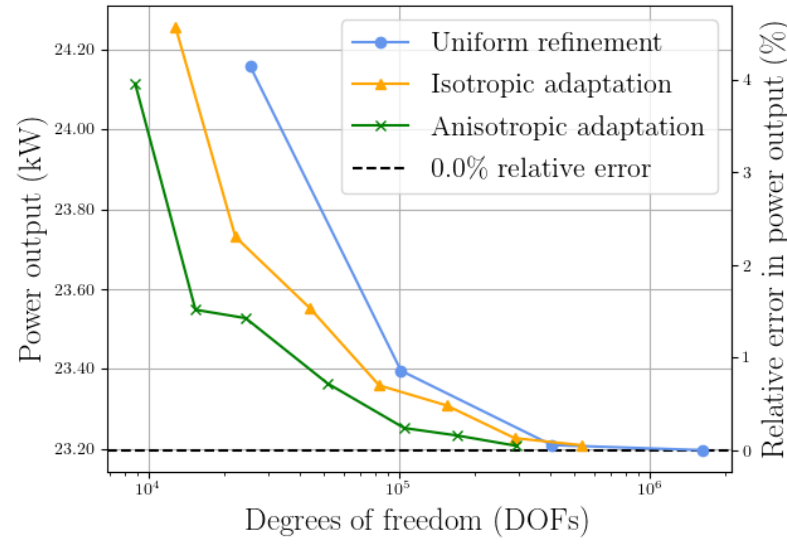

(b) Offset configuration

Figure 2: QoI convergence analysis under uniform refinement, isotropic adaptation and anisotropic adaptation for (a) the aligned and (b) the offset array configurations.

Under both configurations both the isotropic and anisotropic goal-oriented mesh adaptation approaches enable convergence to the benchmark power output values. In each case, the anisotropic approach converges to the benchmark power output using fewer DOFs than the isotropic approach. Except in the case where all three approaches are close to convergence, the goal-oriented strategies achieve the same error levels as uniform refinement using fewer degrees of freedom.

\section{Conclusion}

The main achievement of this paper is the formulation of a goal-oriented error estimate for the nonlinear shallow water equations solved using a mixed discontinuous/continuous finite element method, along with the implementation of isotropic and anisotropic mesh adaptation algorithms using this estimate.

Convergence analysis is performed in the context of the power output of a steady-state tidal turbine problem. This analysis illustrates that far fewer DOFs are required to achieve a certain QoI error threshold using goal-oriented approaches than uniform refinement.

In future work, we intend to use the goal-oriented adaptation framework discussed in this paper for modelling proposed tidal farms, with the aim of accurately approximating the associated power output.

\section{Acknowledgements}

Thank you to the Applied Modelling and Computation Group (AMCG) at Imperial College London, the Firedrake and Thetis development teams and staff and students of the Mathematics of Planet Earth Centre for Doctoral Training (MPE CDT). This work was funded by the Engineering and Physical Sciences Research Council (EPSRC) under grants EP/L016613/1 and EP/R029423/1.

\section{References}

[1] T. Kärnä, S. C. Kramer, L. Mitchell, D. A. Ham, M. D. Piggott, and A. M. Baptista, "Thetis coastal ocean model: discontinuous Galerkin discretization for the three-dimensional hydrostatic equations," Geosci Model Dev, vol. 11, no. 11, pp. 4359-4382, 2018.

[2] F. Rathgeber, D. A. Ham, L. Mitchell, M. Lange, F. Luporini, A. T. McRae, G.-T. Bercea, G. R. Markall, and P. H. Kelly, "Firedrake: automating the finite element method by composing abstractions," ACM T Math Software, vol. 43, no. 3, p. 24, 2016.

[3] C. V. M. Vouriot, A. Angeloudis, S. C. Kramer, and M. D. Piggott, "Fate of large-scale vortices in idealized tidal lagoons," Environmental Fluid Mechanics, vol. 19, pp. 329-348, Apr 2019. 
[4] W. Pan, S. C. Kramer, and M. D. Piggott, "Multi-layer non-hydrostatic free surface modelling using the discontinuous Galerkin method," Ocean Modelling, vol. 134, pp. 68 - 83, 2019.

[5] P. George, F. Hecht, and M. Vallet, "Creation of internal points in Voronoi's type method. Control and adaptation," Adv Eng Software, vol. 13, no. 5-6, pp. 303-312, 1991.

[6] M. Piggott, P. Farrell, C. Wilson, G. Gorman, and C. Pain, "Anisotropic mesh adaptivity for multi-scale ocean modelling," Philosophical Transactions of the Royal Society A: Mathematical, Physical and Engineering Sciences, vol. 367, no. 1907, pp. 4591-4611, 2009.

[7] R. Becker and R. Rannacher, A feed-back approach to error control in finite element methods: Basic analysis and examples. IWR, 1996.

[8] R. Becker and R. Rannacher, "An optimal control approach to a posteriori error estimation in finite element methods," Acta numerica, vol. 10, pp. 1-102, 2001.

[9] P. Houston, E. H. Georgoulis, and E. Hall, "Adaptivity and a posteriori error estimation for DG methods on anisotropic meshes," in Intl Conf on Boundary and Interior Layers, 2006.

[10] V. Dolejší and F. Roskovec, "Goal-oriented error estimates including algebraic errors in discontinuous Galerkin discretizations of linear boundary value problems," Appl Math, vol. 62, no. 6, pp. 579-605, 2017.

[11] P. Power, C. C. Pain, M. Piggott, F. Fang, G. J. Gorman, A. Umpleby, A. J. Goddard, and I. Navon, "Adjoint a posteriori error measures for anisotropic mesh optimisation," Comput Math Appl, vol. 52, no. 8-9, pp. 1213-1242, 2006.

[12] A. Loseille, A. Dervieux, and F. Alauzet, "Fully anisotropic goal-oriented mesh adaptation for 3D steady euler equations," J Comput Phys, vol. 229, no. 8, pp. 2866-2897, 2010.

[13] J. Carpio, J. L. Prieto, and R. Bermejo, "Anisotropic "goal-oriented" mesh adaptivity for elliptic problems," SIAM J Sci Comput, vol. 35, no. 2, pp. A861-A885, 2013.

[14] C. Pain, A. Umpleby, C. De Oliveira, and A. Goddard, "Tetrahedral mesh optimisation and adaptivity for steadystate and transient finite element calculations," Comput Method Appl M, vol. 190, no. 29-30, pp. 3771-3796, 2001.

[15] N. Barral, Time-accurate anisotropic mesh adaptation for three-dimensional moving mesh problems. $\mathrm{PhD}$ thesis, Université Pierre et Marie Curie, 2015.

[16] G. Rokos and G. Gorman, "Pragmatic-parallel anisotropic adaptive mesh toolkit," in Facing the MulticoreChallenge III, pp. 143-144, Springer, 2013.

[17] J. G. Wallwork, N. Barral, D. A. Ham, and M. D. Piggott, "Anisotropic goal-oriented mesh adaptation in Firedrake," in 28th Intl Meshing Roundtable, 2019. (to appear).

[18] C. J. Cotter, D. A. Ham, and C. C. Pain, "A mixed discontinuous/continuous finite element pair for shallow-water ocean modelling," Ocean Model, vol. 26, no. 1-2, pp. 86-90, 2009.

[19] P. D. Lax, "Weak solutions of nonlinear hyperbolic equations and their numerical computation," Communications on Pure and Applied Mathematics, vol. 7, no. 1, pp. 159-193, 1954.

[20] Y. Epshteyn and B. Rivière, "Estimation of penalty parameters for symmetric interior penalty Galerkin methods," J Comput Appl Math, vol. 206, no. 2, pp. 843-872, 2007.

[21] N. Barral, M. G. Knepley, M. Lange, M. D. Piggott, and G. J. Gorman, "Anisotropic mesh adaptation in Firedrake with PETSc DMPlex," in 25th Intl Meshing Roundtable, 2016.

[22] S. W. Funke, P. E. Farrell, and M. Piggott, “Tidal turbine array optimisation using the adjoint approach," Renewable Energy, vol. 63, pp. 658-673, 2014.

[23] P. E. Farrell, D. A. Ham, S. W. Funke, and M. E. Rognes, "Automated derivation of the adjoint of high-level transient finite element programs," SIAM Journal on Scientific Computing, vol. 35, no. 4, pp. C369-C393, 2013.

[24] M. S. Alnæs, A. Logg, K. B. Ølgaard, M. E. Rognes, and G. N. Wells, "Unified form language: A domain-specific language for weak formulations of partial differential equations," ACM T Math Software, vol. 40, no. 2, p. 9, 2014.

[25] "Software used in 'Goal-Oriented Error Estimation and Mesh Adaptation for Shallow Water Modelling'," Dec. 2019.

[26] T. Kärnä, S. Kramer, L. Mitchell, A. Angeloudis, D. A. Ham, J. G. Wallwork, N. Barral, S. Warder, M. Clare, and A. T. T. McRae, "thetisproject/thetis: Thetis coastal ocean model," Feb. 2020.

[27] J. G. Wallwork and M. Clare, "Simulation Code for "Goal-Oriented Error Estimation and Mesh Adaptation for Shallow Water Modelling'," Apr. 2020. 
[28] S. P. Neill, A. Angeloudis, P. E. Robins, I. Walkington, S. L. Ward, I. Masters, M. J. Lewis, M. Piano, A. Avdis, M. D. Piggott, et al., "Tidal range energy resource and optimization-past perspectives and future challenges," Renewable Energy, vol. 127, pp. 763-778, 2018.

[29] D. M. Culley, S. W. Funke, S. C. Kramer, and M. D. Piggott, "Integration of cost modelling within the micro-siting design optimisation of tidal turbine arrays," Renewable Energy, vol. 85, pp. 215 - 227, 2016.

[30] R. J. du Feu, S. W. Funke, S. C. Kramer, J. Hill, and M. D. Piggott, "The trade-off between tidal-turbine array yield and environmental impact: A habitat suitability modelling approach," Renewable Energy, vol. 143, pp. 390 403, 2019.

[31] S. C. Kramer and M. D. Piggott, "A correction to the enhanced bottom drag parameterisation of tidal turbines," Renewable Energy, vol. 92, pp. 385-396, 2016.

[32] C. Geuzaine and J.-F. Remacle, "Gmsh: A 3-d finite element mesh generator with built-in pre-and post-processing facilities," Int J Numer Meth Eng, vol. 79, no. 11, pp. 1309-1331, 2009.

[33] S. Balay, S. Abhyankar, M. F. Adams, J. Brown, P. Brune, K. Buschelman, L. Dalcin, A. Dener, V. Eijkhout, W. D. Gropp, D. Karpeyev, D. Kaushik, M. G. Knepley, D. A. May, L. C. McInnes, R. T. Mills, T. Munson, K. Rupp, P. Sanan, B. F. Smith, S. Zampini, H. Zhang, and H. Zhang, "PETSc users manual," Tech. Rep. ANL-95/11 Revision 3.12, Argonne National Laboratory, 2019.

[34] S. Balay, W. D. Gropp, L. C. McInnes, and B. F. Smith, "Efficient management of parallelism in object oriented numerical software libraries," in Modern Software Tools in Scientific Computing (E. Arge, A. M. Bruaset, and H. P. Langtangen, eds.), pp. 163-202, Birkhäuser Press, 1997.

[35] P. R. Amestoy, I. S. Duff, J.-Y. L'Excellent, and J. Koster, "A fully asynchronous multifrontal solver using distributed dynamic scheduling," SIAM J Matrix Anal A, vol. 23, no. 1, pp. 15-41, 2001.

[36] P. R. Amestoy, A. Guermouche, J.-Y. L'Excellent, and S. Pralet, "Hybrid scheduling for the parallel solution of linear systems," Parallel Comput, vol. 32, no. 2, pp. 136-156, 2006. 